\title{
Testing the Dipole Modulation Model in CMBR
}

\author{
Pranati K. Rath ${ }^{a}$ Pankaj Jain ${ }^{a}$ \\ ${ }^{a}$ Dept. of Physics, Indian Institue of Technology Kanpur, Kanpur - 208016, India \\ E-mail: pranati@iitk.ac.in, pkjain@iitk.ac.in
}

\begin{abstract}
The hemispherical power asymmetry, observed in the CMBR data, has generally been interpreted in terms of the dipole modulation model for the temperature fluctuations. Here we point out that this model leads to several predictions, which can be directly tested in the current data. We suggest tests of the hemispherical power asymmetry both in real and multipole space. We find a significant signal of the dipole modulation model in WMAP and PLANCK data with our tests. The dipole amplitude and direction also agrees, within errors, with earlier results based on hemispherical analysis in multipole space. We also find evidence that the effective dipole modulation amplitude increases with the multipole $l$ in the range $l=2-64$.
\end{abstract}

Keywords: CMBR theory, CMBR data analysis

ArXiv ePrint: 1308.0924 


\section{Contents}

1 Introduction $\quad 1$

2 Tests of the Dipole Modulation Model $r$

2.1 Tests in real space $r$

2.2 Tests in multipole space 3

2.2.1 Correlation Coefficient 4

3 Results 4

$\begin{array}{lll}3.1 & \text { Dipole amplitude in real space } & 5\end{array}$

$\begin{array}{lll}3.2 & \text { Hemispherical power anisotropy in real space } & 7\end{array}$

$\begin{array}{lll}3.3 & \text { Correlations in multipole space } & 8\end{array}$

4 Dependence of dipole amplitude on $l \quad 9$

5 Discussion and Conclusions $r$

\section{Introduction}

At present there exists considerable evidence which indicates that the Universe may not be isotropic on large distance scales. For example, there are several observations which suggest a preferred axis pointing roughly towards Virgo [1-6]. One also observes a hemispherical anisotropy [7-12], where the power extracted from two different hemispheres shows significant difference from one another. The power in each hemisphere is estimated by making a harmonic decomposition of the masked sky. The significance of anisotropy is found to be about 3 sigma [13] with the axis of maximum asymmetry having $(\theta, \phi)$ pointing towards $\left(115^{\circ}, 227^{\circ}\right)$ in galactic coordinates. This direction is nearly perpendicular to the direction towards Virgo. These observations suggest a violation of the cosmological principle and might indicate that one needs a revision of the Big Bang cosmology. There exist many models which propose to address these issues. It has been suggested that the anisotropic modes, generated during the pre-inflationary phase might re-enter the horizon before the current time [14-16]. Hence these might explain the observed anisotropy even within the framework of the inflationary Big Bang model.

Theoretically, the observed hemispherical asymmetry in CMBR is assumed to arise from the model [17-20],

$$
\triangle T(\hat{n})=g(\hat{n})(1+A \hat{\lambda} \cdot \hat{n})
$$

where $\triangle T(\hat{n})$ is the measured temperature fluctuation in the direction $\hat{n}, g(\hat{n})$ a statistically isotropic field, $A$ the dipole amplitude and $\hat{\lambda}$ the preferred direction. Taking the preferred direction along the z-direction, the temperature modulated field can be written as,

$$
\triangle T(\hat{n})=g(\hat{n})(1+A \cos \theta)
$$

The extracted value of the dipole modulation amplitude from CMBR data is given by [7-12], $A=$ $0.072 \pm 0.022$. The precise value of $A$ shows some dependence on the input CMBR map. However the difference is relatively small and not relevant for our analysis. It is likely that this model may not extend to all values of $l$. The true anisotropy model is likely to be more complicated [21-23]. Here we discuss several implications of this model and test them in the observed CMBR data. These provide additional tests of this model. 


\section{Tests of the Dipole Modulation Model}

In this section we suggest several tests of the dipole modulation model, both in pixel and multipole space. The temperature field, $\Delta T(\hat{n})$ can be analyzed by making a spherical harmonic decomposition,

$$
\triangle T(\hat{n})=\sum_{l=1}^{\infty} \sum_{m=-l}^{+l} a_{l m} Y_{l}^{m}(\hat{n})
$$

Similarly, we expand the statistically isotropic field, $g(\hat{n})$,

$$
g(\hat{n})=\sum_{l, m} \tilde{a}_{l m} Y_{l}^{m}(\hat{n})
$$

The expansion coefficients, $\tilde{a}_{l m}$, in this case are different from $a_{l m}$ due to the presence of dipole modulation in $\Delta T$. However, the multipole power, $C_{l}$, is same for both the fields, $\triangle T(\hat{n})$ and $g(\hat{n})$. We shall show this explicitly in Section 2.2. We next describe our tests of the dipole modulation in real space, followed by tests in multipole space.

\subsection{Tests in real space}

Let us consider the observed squared temperature fluctuation field,

$$
f(\theta, \phi)=(\Delta T(\theta, \phi))^{2}
$$

where we have expressed the unit vector, $\hat{n}$, in terms of the spherical polar coordinates, $(\theta, \phi)$. If the dipole modulation model, Eq. 1.1, provides an accurate description of the real data, we expect that $f(\theta, \phi)$ can be expressed as,

$$
f(\theta, \phi) \approx g(\theta, \phi) g^{*}(\theta, \phi)(1+2 A \hat{\lambda} \cdot \hat{n})
$$

where on the right hand side we have only kept terms linear in the dipole amplitude $A$. Using Eq. 2.2 and taking the ensemble average, we obtain,

$$
\langle f(\theta, \phi)\rangle \approx \sum_{l, m} \sum_{l^{\prime} m^{\prime}}\left\langle\tilde{a}_{l m} \tilde{a}_{l^{\prime} m^{\prime}}^{*}\right\rangle Y_{l}^{m}(\theta, \phi) Y_{l^{\prime}}^{m^{\prime} *}(\theta, \phi)(1+2 A \hat{\lambda} \cdot \hat{n})
$$

Using

$$
\left\langle\tilde{a}_{l m} \tilde{a}_{l^{\prime} m^{\prime}}^{*}\right\rangle=\delta_{l l^{\prime}} \delta_{m m^{\prime}} C_{l}
$$

and the sum

$$
\sum_{m} Y_{l}^{m}(\theta, \phi) Y_{l}^{m *}(\theta, \phi)=\frac{2 l+1}{4 \pi}
$$

we obtain,

$$
\langle f(\theta, \phi)\rangle \approx \sum_{l} \frac{2 l+1}{4 \pi} C_{l}(1+2 A \hat{\lambda} \cdot \hat{n})
$$

Hence if we expand the observed temperature square field, $f(\theta, \phi)$, in spherical harmonics,

$$
f(\theta, \phi)=\sum_{l m} A_{l m} Y_{l}^{m}(\theta, \phi) .
$$

we should find a significant dipole contribution. The amplitude of the dipole would be related to $A$ with the direction equal to $\hat{\lambda}$. This observable, therefore, provides a simple test of the dipole modulation model. 
We also define another observable, $Q$, which sums temperature fluctuation square in a particular hemisphere. Let $N$ represent the total number of pixels in any particular hemisphere of the real sky. We define,

$$
Q=\frac{1}{N} \sum_{i=1}^{N} f(\theta, \phi)
$$

We divide the sky into an upper and lower hemisphere, along the chosen $z$ direction. The resulting values of $Q$ in the two hemispheres are denoted as $Q_{+}$and $Q_{-}$. We define the quantity, $S_{Q}$, such that,

$$
S_{Q}=\frac{Q_{+}}{Q_{-}}
$$

This is maximized over all possible choices of the $z$ axis. The maximum value of $S_{Q}$ provides another useful statistic to test for hemispherical anisotropy in real space. The corresponding $z$ axis gives an estimate of the dipole modulation axis $\hat{\lambda}$. We should point out that using this procedure we will also get contributions from higher order odd multipoles if they are present in the harmonic expansion, Eq. 2.8 , of $f(\theta, \phi)$. Hence the results obtained by this procedure would, in general, differ from the dipole extracted by making a harmonic decomposition of $f(\theta, \phi)$.

\subsection{Tests in multipole space}

The two point correlations of the isotropic temperature field, $g(\hat{n})$, in two different directions, $\hat{n}$ and $\hat{n}^{\prime}$, are given as,

$$
\left\langle g(\hat{n}) g^{*}\left(\hat{n}^{\prime}\right)\right\rangle=\sum_{l m} C_{l} Y_{l}^{m}(\hat{n}) Y_{l}^{m *}\left(\hat{n}^{\prime}\right)
$$

where $C_{l}$ is the angular power spectrum. The two point correlations of the dipole modulated temperature field, Eq. 1.1, can be expressed as,

$$
\left\langle\Delta T(\hat{n}) \Delta T^{*}\left(\hat{n}^{\prime}\right)\right\rangle=\sum_{l m} C_{l} Y_{l}^{m}(\hat{n}) Y_{l}^{m *}\left(\hat{n}^{\prime}\right)\left[1+A \cos \theta+A \cos \theta^{\prime}\right]
$$

where we drop terms quadratic in $A$. The two point correlation function of the spherical harmonic coefficients $a_{l m}$, defined in Eq. 2.1, can be expressed as,

$$
\left\langle a_{l m} a_{l^{\prime} m^{\prime}}^{*}\right\rangle=\int d \Omega_{\hat{n}} d \Omega_{\hat{n}^{\prime}} Y_{l}^{m *}(\hat{n}) Y_{l^{\prime}}^{m^{\prime}}\left(\hat{n}^{\prime}\right)\left\langle\triangle T(\hat{n}) \triangle T\left(\hat{n}^{\prime}\right)\right\rangle
$$

Using Eq. 2.13, we obtain,

$$
\left\langle a_{l m} a_{l^{\prime} m^{\prime}}^{*}\right\rangle=\left\langle a_{l m} a_{l^{\prime} m^{\prime}}^{*}\right\rangle_{i s o}+\left\langle a_{l m} a_{l^{\prime} m^{\prime}}^{*}\right\rangle_{d m}
$$

where $\left\langle a_{l m} a_{l^{\prime} m^{\prime}}^{*}\right\rangle_{i s o}=C_{l} \delta_{l l^{\prime}} \delta_{m m^{\prime}}$, and

$$
\left\langle a_{l m} a_{l^{\prime} m^{\prime}}^{*}\right\rangle_{d m}=A C_{l^{\prime}} \xi_{l m ; l^{\prime} m^{\prime}}^{0}+A C_{l} \xi_{l m ; l^{\prime} m^{\prime}}^{0}
$$

where,

$$
\begin{aligned}
\xi_{l m ; l^{\prime} m^{\prime}}^{0}= & \int d \Omega Y_{l}^{m *}(\hat{n}) Y_{l^{\prime}}^{m^{\prime}}(\hat{n}) \cos \theta \\
= & \delta_{m^{\prime}, m}\left[\sqrt{\frac{(l-m+1)(l+m+1)}{(2 l+1)(2 l+3)}} \delta_{l^{\prime}, l+1}\right. \\
& \left.+\sqrt{\frac{(l-m)(l+m)}{(2 l+1)(2 l-1)}} \delta_{l^{\prime}, l-1}\right]
\end{aligned}
$$

For $l^{\prime}=l+1$,

$$
\left\langle a_{l m} a_{l^{\prime} m^{\prime}}^{*}\right\rangle=A\left(C_{l+1}+C_{l}\right) \delta_{m^{\prime}, m}\left[\sqrt{\frac{(l-m+1)(l+m+1)}{(2 l+1)(2 l+3)}} \delta_{l^{\prime}, l+1}\right]
$$


and for $l^{\prime}=l-1$,

$$
\left\langle a_{l m} a_{l^{\prime} m^{\prime}}^{*}\right\rangle=A\left(C_{l-1}+C_{l}\right) \delta_{m^{\prime}, m}\left[\sqrt{\frac{(l-m)(l+m)}{(2 l+1)(2 l-1)}} \delta_{l^{\prime}, l-1}\right]
$$

Hence the dipole modulation model predicts a correlation between $a_{l, m}$ and $a_{l+1, m}$. We also find that the dipole modulation term does not give any contribution to the power $\left\langle a_{l m} a_{l m}^{*}\right\rangle$ if we only retain terms linear in $A$. Hence the power, $C_{l}$, of the field $\Delta T(\hat{n})$ is same as that of $g(\hat{n})$. The correlation in Eq. 2.19 may be positive or negative, depending on the sign of $A$. However we expect that all the multipoles would be correlated with the same sign. We can test for this correlation in real data by defining,

$$
C_{l, l+1}=\frac{l(l+1)}{2 l+1} \sum_{m=-l}^{l} a_{l m} a_{l+1, m}^{*}
$$

The sum over $m$ contains $(2 l+1)$ terms. After dividing by $(2 l+1)$ we obtain an average value of the correlation for each multipole. The normalization factor $l(l+1)$ is inserted in order to make it consistent with the measure $l(l+1) C_{l}$ used in [7-12]. We do not find a significant signal of anisotropy if the prefactor $l(l+1) /(2 l+1)$ is not used in the definition of $C_{l, l+1}$. The sum of $C_{l, l+1}$ over a chosen range of multipoles defines our statistic, $S_{H}(L)$,

$$
S_{H}(L)=\sum_{l=2}^{L} C_{l, l+1}
$$

\subsubsection{Correlation Coefficient}

The correlation of the multipole coefficients, $a_{l m}$, using the dipole modulation temperature field is given by Eq. 2.18. We can test this dependence by computing the Pearson correlation coefficient, $r$, between $x$ and $y$, defined as,

$$
\begin{aligned}
y & =\sum_{m} a_{l m} a_{l+1, m}^{*} \\
x & =\sum_{m}\left(C_{l+1}+C_{l}\right) \sqrt{\frac{(l-m+1)(l+m+1)}{(2 l+1)(2 l+3)}}
\end{aligned}
$$

These are defined by summing both sides in Eq. 2.18 over $m$ and setting $l^{\prime}=l+1$. Eq. 2.18 implies that,

$$
y=A x
$$

We compute the linear correlation coefficient to see whether there exists a linear correlation between these two variables over a certain range of $l$ values. We again search over all directions in order to maximize the correlation, $r$.

\section{Results}

In this section we present results for dipole modulation using the tests in real and multipole space proposed above. The significance of anisotropy is determined by comparing the result for real data with that corresponding to 4000 random samples. The quoted P-values represent the probability that the dipole extracted from real data may arise as a random fluctuation from a statistically isotropic sample. We use the WMAP 9 year ILC map as well as the PLANCK [24] NILC, SMICA and SEVEM maps for our analysis.

In most of our analysis we use masked sky in order to eliminate the region close to galactic plane, which has very strong foreground contamination. We use the KQ85 mask for the WMAP-ILC map and the COM-MASK-gal-07 mask for the PLANCK maps. The masked regions are filled by randomly generated isotropic data. The random data is obtained by generating a full sky, random, isotropic, 
high resolution map with $N_{\text {side }}=512$. After applying the inverse mask to this map, it is added to the masked real map at the same resolution. The resulting map is downgraded to $N_{\text {side }}=32$. This corresponds to maximum $l$ value of 64 , for which significant signal of hemispherical anisotropy has been observed [13]. The mask boundaries get smoothed when we downgrade the map and hence this procedure eliminates any breaks that might have arisen if we worked directly with the low resolution map. We also test the sensitivity of our results to mask boundaries by using an alternative procedure. The PLANCK temperature and the mask maps are provided at $N_{\text {side }}=2048$. We generate the full sky map at this resolution. This map is downgraded to $N_{\text {side }}=256$ after smoothing with a Gaussian beam having FWHM equal to three times of the pixel size of the low resolution map. This map is then downgraded to $N_{\text {side }}=32$. This procedure gave results very close to the one described earlier.

The results are found to depend significantly on the random realization used to fill the masked regions. Hence, the results for dipole power and direction are obtained after averaging the results of 100 maps, which are generated by filling the masked regions by different random samples.

\subsection{Dipole amplitude in real space}

We first consider the dipole extracted by making the spherical harmonic decomposition, Eq. 2.9, of the temperature square field. If the field is statistically isotropic, none of the multipoles would be significantly different from those corresponding to a random realization. If the dipole modulation is present, then we should detect a significant dipole. Our calculations show that a direct extraction of the dipole power from data does not yield a statistically significant result. The problem is traced to the fact that the temperature square dipole power does not yield a sensitive probe of the dipole modulation model. We check the sensitivity of this measure by direct simulations. We generate a random isotropic realization of the CMB temperature map and multiply it with the dipole modulation factor, setting $A=0.072$. This generates a particular realization of an anisotropic map. The significance of the measure is determined by comparison with isotropic random samples, as explained above. We do not find a statistically significant signal for $A=0.072$.

The reason for this lack of sensitivity is easily understood. The statistical measure used in [7-12] is the sum of $l(l+1) C_{l}$ over $l$. However in our case the dipole amplitude of the temperature square field, Eq. 2.8, essentially sums over $(2 l+1) C_{l}$ and hence puts lower weight on higher multipoles. We, therefore, define an alternate measure, by removing some of the low $l$ multipoles of the temperature field. We consider two cuts:

(a) remove $l=2-4$ multipoles from the temperature field.

(b) remove $l=2-8$ multipoles from the temperature field.

The resulting dipole power of the temperature square field, $f(\theta, \phi)$, for the two cuts (a) and (b) is denoted as, $\mathcal{C}_{1}(4)$ and $\mathcal{C}_{1}(8)$ respectively. The dipole power and the corresponding direction parameters, $(\theta, \phi)$, are given in Tables 1 and 2 for $\mathcal{C}_{1}(4)$ and $\mathcal{C}_{1}(8)$ respectively. Here we extract these parameters using the full sky map, i.e. without masking the galactic plane. The significance of the dipole is given in terms of P-values, defined above. In Fig. 1, we show the distribution of the dipole power, $\mathcal{C}_{1}(8)$, for isotropic random samples.

We next present results obtained by using masked sky analysis. In Tables 3 and 4, we present the results for $\mathcal{C}_{1}(4)$, cut (a), and $\mathcal{C}_{1}(8)$, cut (b), respectively, along with the corresponding direction parameters and $\mathrm{P}$-values. We find a signal of anisotropy with significance ranging from $2-3$ sigmas. As explained above, the results given in Tables 3 and 4 are obtained after taking a mean over 100 different realizations. The variation of the axes for these 100 realizations for SMICA using cut (b) is shown in Fig. 2. The mean values of $(\theta, \phi)$ in this case are found to be $\left(130^{\circ}, 244^{\circ}\right)$ and the corresponding standard deviations $\left(11^{\circ}, 23^{\circ}\right)$. Hence we find that our extracted axis parameters are consistent with those found in [7-12]. Similar spread in axes parameters are found with other maps.

We also simulate the dipole modulated field by taking the dipole amplitude $A=0.072 \pm 0.022$ and extract the power, $\mathcal{C}_{1}$. For the case of masked sky, we find that the dipole amplitude, $\mathcal{C}_{1}(4)=$ $(3.3 \pm 1.1) \times 10^{-7}\left(\mathrm{mK}^{4}\right)$ and $\mathcal{C}_{1}(8)=(1.4 \pm 0.7) \times 10^{-7}\left(\mathrm{mK}^{4}\right)$. Here the error arises due to the error in the extracted dipole amplitude, $|\Delta A|=0.022$. Hence we find that the theoretical expectations 


\begin{tabular}{|c|c|c|c|}
\hline & $\mathcal{C}_{1}(4)\left(\mathrm{mK}^{4}\right)$ & $(\theta, \phi)$ & P-value \\
\hline NILC & $5.11 \times 10^{-7}$ & $\left(130^{\circ}, 230^{\circ}\right)$ & $2.92 \%$ \\
\hline SMICA & $6.96 \times 10^{-7}$ & $\left(122^{\circ}, 215^{\circ}\right)$ & $0.82 \%$ \\
\hline SEVEM & $4.17 \times 10^{-7}$ & $\left(132^{\circ}, 237^{\circ}\right)$ & $5.82 \%$ \\
\hline WMAP-ILC & $7.42 \times 10^{-7}$ & $\left(123^{\circ}, 213^{\circ}\right)$ & $0.57 \%$ \\
\hline
\end{tabular}

Table 1. The extracted dipole power of the temperature square field and the corresponding direction parameters, for different maps, using $N_{\text {side }}=32$, without masking the galactic plane. Here we have imposed cut (a) on the temperature field.

\begin{tabular}{|c|c|c|c|}
\hline & $\mathcal{C}_{1}(8)\left(\mathrm{mK}^{4}\right)$ & $(\theta, \phi)$ & P-value \\
\hline NILC & $1.43 \times 10^{-7}$ & $\left(124^{\circ}, 287^{\circ}\right)$ & $4.05 \%$ \\
\hline SMICA & $1.42 \times 10^{-7}$ & $\left(125^{\circ}, 236^{\circ}\right)$ & $4.25 \%$ \\
\hline SEVEM & $1.51 \times 10^{-7}$ & $\left(127^{\circ}, 285^{\circ}\right)$ & $3.45 \%$ \\
\hline WMAP-ILC & $1.21 \times 10^{-7}$ & $\left(131^{\circ}, 241^{\circ}\right)$ & $5.77 \%$ \\
\hline
\end{tabular}

Table 2. The extracted dipole power of the temperature square field and the corresponding direction parameters for cut (b), using $N_{\text {side }}=32$, without masking the galactic plane.

\begin{tabular}{|c|c|c|c|}
\hline & $\mathcal{C}_{1}(4)\left(\mathrm{mK}^{4}\right)$ & $(\theta, \phi)$ & P-value \\
\hline NILC & $4.07 \times 10^{-7}\left(2.91 \times 10^{-7}\right)$ & $\left(135^{\circ}, 226^{\circ}\right)$ & $6.05 \%$ \\
\hline SMICA & $4.15 \times 10^{-7}\left(3.01 \times 10^{-7}\right)$ & $\left(135^{\circ}, 224^{\circ}\right)$ & $5.85 \%$ \\
\hline SEVEM & $3.93 \times 10^{-7}\left(2.80 \times 10^{-7}\right)$ & $\left(136^{\circ}, 229^{\circ}\right)$ & $6.82 \%$ \\
\hline WMAP-ILC & $4.06 \times 10^{-7}\left(2.28 \times 10^{-7}\right)$ & $\left(131^{\circ}, 216^{\circ}\right)$ & $6.65 \%$ \\
\hline
\end{tabular}

Table 3. The extracted dipole power of the temperature square field and the direction parameters for different maps, using masked sky analysis with $N_{\text {side }}=32$, for cut (a). The $\mathcal{C}_{1}$ values are obtained after taking a mean of 100 different realizations, with the masked regions filled by random isotropic data. The corresponding standard deviations are given in brackets.

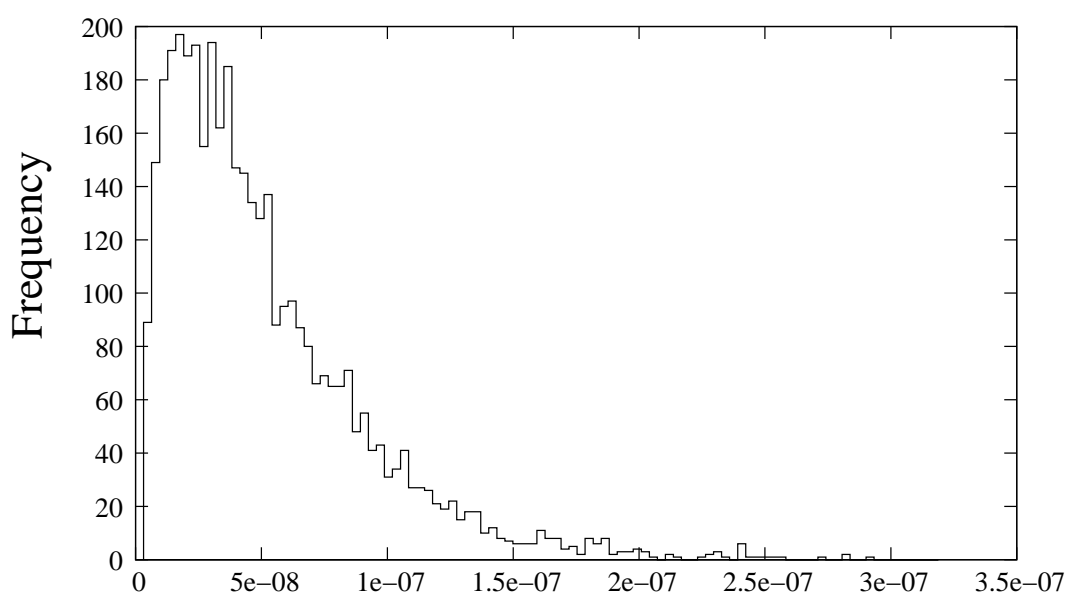

Dipole power $\left(\mathrm{mK}^{4}\right)$

Figure 1. The distribution of the dipole power, $\mathcal{C}_{1}(8)\left(\mathrm{mK}^{4}\right)$, of the temperature square field for isotropic random realizations using cut (b).

agree, within errors, with the extracted dipole power from data. Our results suggest that there exists a significant signal for the dipole modulation model, Eq. 1.1, in the CMBR data. 


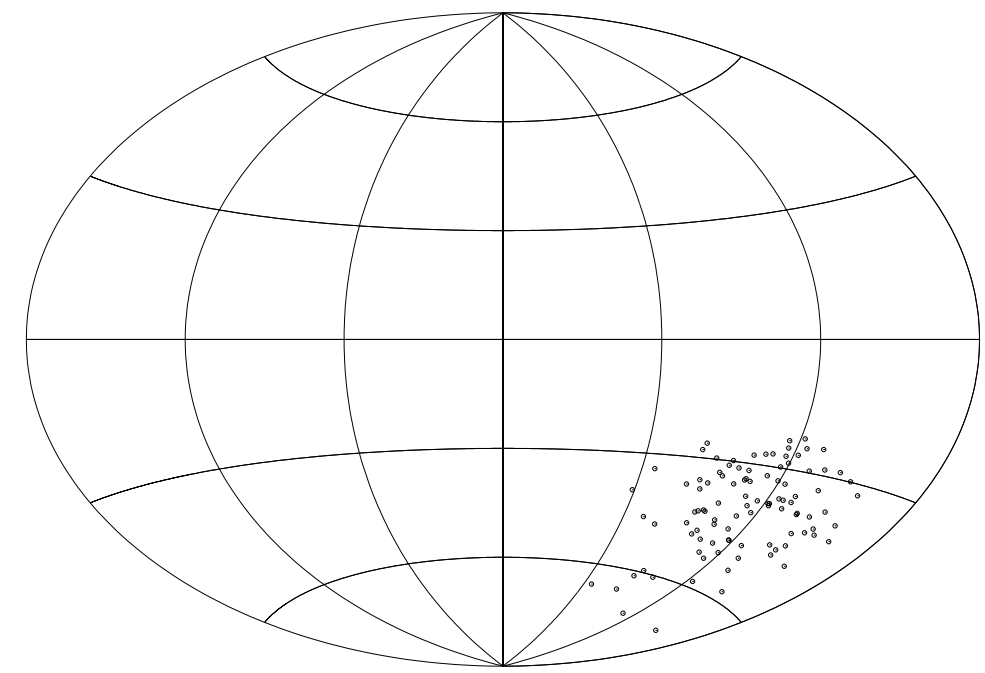

Figure 2. The dipole axes parameters extracted from the PLANCK SMICA map using cut (b). The different axes correspond to different random fillings of the masked regions.

\begin{tabular}{|c|c|c|c|}
\hline & $\mathcal{C}_{1}(8)\left(\mathrm{mK}^{4}\right)$ & $(\theta, \phi)$ & P-value \\
\hline NILC & $2.04 \times 10^{-7}\left(1.15 \times 10^{-7}\right)$ & $\left(130^{\circ}, 245^{\circ}\right)$ & $0.92 \%$ \\
\hline SMICA & $2.06 \times 10^{-7}\left(1.16 \times 10^{-7}\right)$ & $\left(130^{\circ}, 244^{\circ}\right)$ & $0.89 \%$ \\
\hline SEVEM & $2.05 \times 10^{-7}\left(1.14 \times 10^{-7}\right)$ & $\left(130^{\circ}, 246^{\circ}\right)$ & $0.92 \%$ \\
\hline WMAP-ILC & $1.8 \times 10^{-7}\left(1.15 \times 10^{-7}\right)$ & $\left(126^{\circ}, 242^{\circ}\right)$ & $1.45 \%$ \\
\hline
\end{tabular}

Table 4. The extracted dipole power of the temperature square field and the direction parameters for cut (b), using $N_{\text {side }}=32$, after masking the galactic plane. The $\mathcal{C}_{1}$ values are obtained after taking a mean of 100 different realizations, with the masked regions filled by random isotropic data. The corresponding standard deviations are given in brackets.

\subsection{Hemispherical power anisotropy in real space}

We next apply our hemispherical power anisotropy test in real space. In this case we compute the power by squaring the temperature fluctuation field in each hemisphere in pixel space. We generate mask files for the upper hemisphere $\left(0<\theta \leq 90^{\circ}\right)$ and the lower hemisphere $\left(\theta>90^{\circ}\right)$. The resulting power obtained after applying these masks is normalized with the number of pixels in the respective hemispheres. The ratio of powers in the two hemispheres gives us an estimate of $S_{Q}$, defined in Eq. 2.11. In this case we present results only after eliminating the contaminated galactic plane by using the masks KQ85 and COM-MASK-gal-07 for WMAP and PLANCK data respectively. The statistic $S_{Q}$ is determined directly from the masked sky.

With this measure also, we do not obtain significant results unless we impose cuts to eliminate a few low multipoles of the temperature field. The results after imposing cuts (a) and (b) are given in Tables 5 and 6 respectively. Here the angle parameters $(\theta, \phi)$ are determined by searching over all possible directions in order to maximize $S_{Q}$. In Tables 5 and 6 , the P-values in brackets are obtained by determining the number of times an isotropic random sample yields an $S_{Q}$ larger than real data. We use 4000 random samples for this purpose. We do not explicitly search over directions for each random sample. Hence the significance obtained by this procedure would provide an overestimate. In order to account for the search, we assume Gaussian statistics, and determine the P-value corresponding to two additional search parameters. This yields the true P-values given in Tables 5 and 6 . These indicate that the significance of dipole anisotropy in this case is about 2 sigma for PLANCK data, a marginally significant result. The WMAP-ILC map yields a much higher significance for cut (a), roughly equal to 3.7 sigmas. We also find that the direction obtained aligns reasonably well with that 
corresponding to hemispherical power asymmetry in multipole space [7-12].

The dipole modulation model with $A=0.072 \pm 0.022$ leads to a maximum $S_{Q}$ value of $1.24 \pm 0.08$, both for cut (a) and (b). Hence the value extracted from PLANCK data for cut (b) agrees with this prediction, within errors. The value corresponding to cut (a) is found to be slightly larger than predicted. The WMAP data, however, gives somewhat larger result. In particular the WMAP result for cut (a) deviates from the predicted value by more than 3 sigmas.

\begin{tabular}{|c|c|c|c|}
\hline & max. $S_{Q}$ & $(\theta, \phi)$ & P-value \\
\hline NILC & 1.36 & $\left(94^{\circ}, 236^{\circ}\right)$ & $5.4 \%(0.57 \%)$ \\
\hline SMICA & 1.35 & $\left(94^{\circ}, 236^{\circ}\right)$ & $6.4 \%(0.7 \%)$ \\
\hline SEVEM & 1.34 & $\left(94^{\circ}, 236^{\circ}\right)$ & $6.4 \%(0.7 \%)$ \\
\hline WMAP-ILC & 1.53 & $\left(135^{\circ}, 202^{\circ}\right)$ & $0.022 \%(0.001 \%)$ \\
\hline
\end{tabular}

Table 5. The extracted values corresponding to the hemispherical power asymmetry with cut (a), after masking the galactic plane, for different maps using $N_{\text {side }}=32$. The P-values in brackets do not account for the search over the axes parameters.

\begin{tabular}{|c|c|c|c|}
\hline & max. $S_{Q}$ & $(\theta, \phi)$ & P-value \\
\hline NILC & 1.27 & $\left(135^{\circ}, 202^{\circ}\right)$ & $5.2 \%(0.55 \%)$ \\
\hline SMICA & 1.27 & $\left(135^{\circ}, 202^{\circ}\right)$ & $4.9 \%(0.50 \%)$ \\
\hline SEVEM & 1.27 & $\left(102^{\circ}, 232^{\circ}\right)$ & $5.0 \%(0.52 \%)$ \\
\hline WMAP-ILC & 1.39 & $\left(135^{\circ}, 202^{\circ}\right)$ & $4.9 \%(0.05 \%)$ \\
\hline
\end{tabular}

Table 6. The extracted values corresponding to the hemispherical power asymmetry for cut (b), after masking the galactic plane, for different maps using $N_{\text {side }}=32$.

\subsection{Correlations in multipole space}

The dipole modulation model leads to correlations among different multipoles, given in Eq. 2.18 or 2.19. We can, therefore, test the model by computing the significance of these correlations in data. We compute the correlation, $C_{l, l+1}$, defined in Eq. 2.20, at a particular $l$ value. The statistic is then obtained by summing over a certain range of $l$ values as in Eq. 2.21. Following the range of observed hemispherical power asymmetry $[13,25]$, we consider the multipole range $l=2-64$. We extract the maximum value of $S_{H}(L)$ for different maps after searching over all possible directions. Note that for the multipole range $l=2-64, L=63$. The true P-values are extracted, as in section 4.2, after accounting for the search over the angle parameters. The results, after masking the galactic plane, are given in Table 7. In this case also the masked regions are filled by random isotropic samples. The results are obtained after averaging over 100 such realizations. The mean and standard deviations of the extracted $S_{H}(L)$ are given in Table 7. For SMICA, the standard deviations of the angle parameters, $(\theta, \phi)$, are $\left(21^{\circ}, 34^{\circ}\right)$. We find a signal of anisotropy with significance approximately equal to 2.4 sigmas. The dipole modulation model, with $A=0.072 \pm 0.022$, yields a value of $S_{H}(L)$ equal to $0.023 \pm 0.008$. Hence in this case also the value extracted from data agrees well with this prediction.

\begin{tabular}{|c|c|c|c|}
\hline & $\max . S_{H}(L)\left(\mathrm{mK}^{2}\right)$ & $(\theta, \phi)$ & P-value \\
\hline NILC & $2.25 \times 10^{-2}\left(0.53 \times 10^{-2}\right)$ & $\left(115^{\circ}, 234^{\circ}\right)$ & $1.5 \%(0.12 \%)$ \\
\hline SMICA & $2.26 \times 10^{-2}\left(0.54 \times 10^{-2}\right)$ & $\left(115^{\circ}, 232^{\circ}\right)$ & $1.5 \%(0.12 \%)$ \\
\hline SEVEM & $2.24 \times 10^{-2}\left(0.53 \times 10^{-2}\right)$ & $\left(114^{\circ}, 234^{\circ}\right)$ & $1.5 \%(0.12 \%)$ \\
\hline WMAP-ILC & $2.22 \times 10^{-2}\left(0.66 \times 10^{-2}\right)$ & $\left(104^{\circ}, 227^{\circ}\right)$ & $1.3 \%(0.10 \%)$ \\
\hline
\end{tabular}

Table 7. The extracted maximum values of $S_{H}(L)$ for different maps using $N_{\text {side }}=32$, using masked sky analysis over the multipole range $l=2-64$. The standard deviations of $S_{H}(L)$ are given in brackets. The $\mathrm{P}$-values given in brackets do not account for the search over the two axes parameters. 
Pearson correlation coefficient: We finally compute the Pearson correlation coefficient, $r$, between variables $x$ and $y$, defined in section 3.1, over the range $l=2-64$, in order to test the relationship, Eq. 2.18. Here again we make a search over all directions in order to maximize the value of $r$. The results for cuts (a) and (b) are given in Tables 8 and 9 respectively. In this case, cut (a) does not yield a significant results whereas cut (b) yields a marginally significant signal. Furthermore, the direction parameters also show larger deviation in comparison to those found in hemispherical multipole space analysis [7-12]. For SMICA, the standard deviations of $(\theta, \phi)$ are found to be $\left(23^{\circ}, 74^{\circ}\right)$, which are quite large in comparison to remaining tests. The dipole modulation model, Eq. 1.1, with $A=0.072 \pm 0.022$ yields the maximum value of $r$ equal to approximately 0.2 . Hence our analysis gives a much larger value in comparison to this prediction. This suggests that this measure may not be a sensitive probe and gets a large contribution due to fluctuations in data. Hence the discrepancy found by this probe is not very significant.

\begin{tabular}{|c|c|c|c|}
\hline & $\max . r$ & $(\theta, \phi)$ & P-value \\
\hline NILC & $0.61(0.11)$ & $\left(163^{\circ}, 219^{\circ}\right)$ & $26 \%(4.4 \%)$ \\
\hline SMICA & $0.61(0.11)$ & $\left(162^{\circ}, 215^{\circ}\right)$ & $25 \%(4.3 \%)$ \\
\hline SEVEM & $0.61(0.11)$ & $\left(164^{\circ}, 229^{\circ}\right)$ & $27 \%(4.7 \%)$ \\
\hline WMAP-ILC & $0.66(0.09)$ & $\left(150^{\circ}, 208^{\circ}\right)$ & $15 \%(2.2 \%)$ \\
\hline
\end{tabular}

Table 8. The extracted maximum values of the correlation coefficient for different maps with cut (a) using $N_{\text {side }}=32$, over the range $l=2-64$, using masked sky analysis. The standard deviation of $r$ is given in brackets.

\begin{tabular}{|c|c|c|c|}
\hline & max. $r$ & $(\theta, \phi)$ & P-value \\
\hline NILC & $0.57(0.10)$ & $\left(130^{\circ}, 261^{\circ}\right)$ & $6.9 \%(0.77 \%)$ \\
\hline SMICA & $0.57(0.10)$ & $\left(130^{\circ}, 261^{\circ}\right)$ & $6.9 \%(0.77 \%)$ \\
\hline SEVEM & $0.57(0.10)$ & $\left(130^{\circ}, 263^{\circ}\right)$ & $6.7 \%(0.75 \%)$ \\
\hline WMAP-ILC & $0.53(0.09)$ & $\left(134^{\circ}, 260^{\circ}\right)$ & $12 \%(1.52 \%)$ \\
\hline
\end{tabular}

Table 9. The extracted maximum values of the correlation coefficient for different maps using cut (b) and $N_{\text {side }}=32$, over the range $l=2-64$, using masked sky analysis. The standard deviation of $r$ is given in brackets.

\section{Dependence of dipole amplitude on $l$}

We next determine how the dipole modulation amplitude $A$ varies with the multipole $l$. For this analysis we extract the effective value of $A$ over a narrow range of $l$ values. These are taken to be, $l=2-8,9-15, \ldots, 58-64$. The extracted dipole power of the temperature square field, using the SMICA map, is shown in Fig. 3. For comparison we also show results for simulated maps corresponding to $A=0.072$ and $A=0.032$. Here we do not show the results for $l=2-8,9-15$ since the fluctuations in this multipole range are found to be very large. Hence it is not possible to make a reliable comparison of theoretical prediction with data. We see from Fig. 3 that the standard value $A=0.072$ gives a good description of the high $l$ multipoles. However the low $l$ multipoles prefer a value closer to $A=0.032$. Hence we find that the effective value of $A$ increases with $l$. A more detailed investigation of this effect is postponed for future research.

\section{Discussion and Conclusions}

We find a significant signal of the dipole modulation model, Eq. 1.1, in data. In real space we propose a measure based on the dipole power of the temperature square field. This measure reveals a significant signal of anisotropy provided we eliminate a few low $l$ multipoles. The extracted signal in this case essentially sums the $C_{l}$ values over $l$ after weighting them with $(2 l+1)$. In comparison 


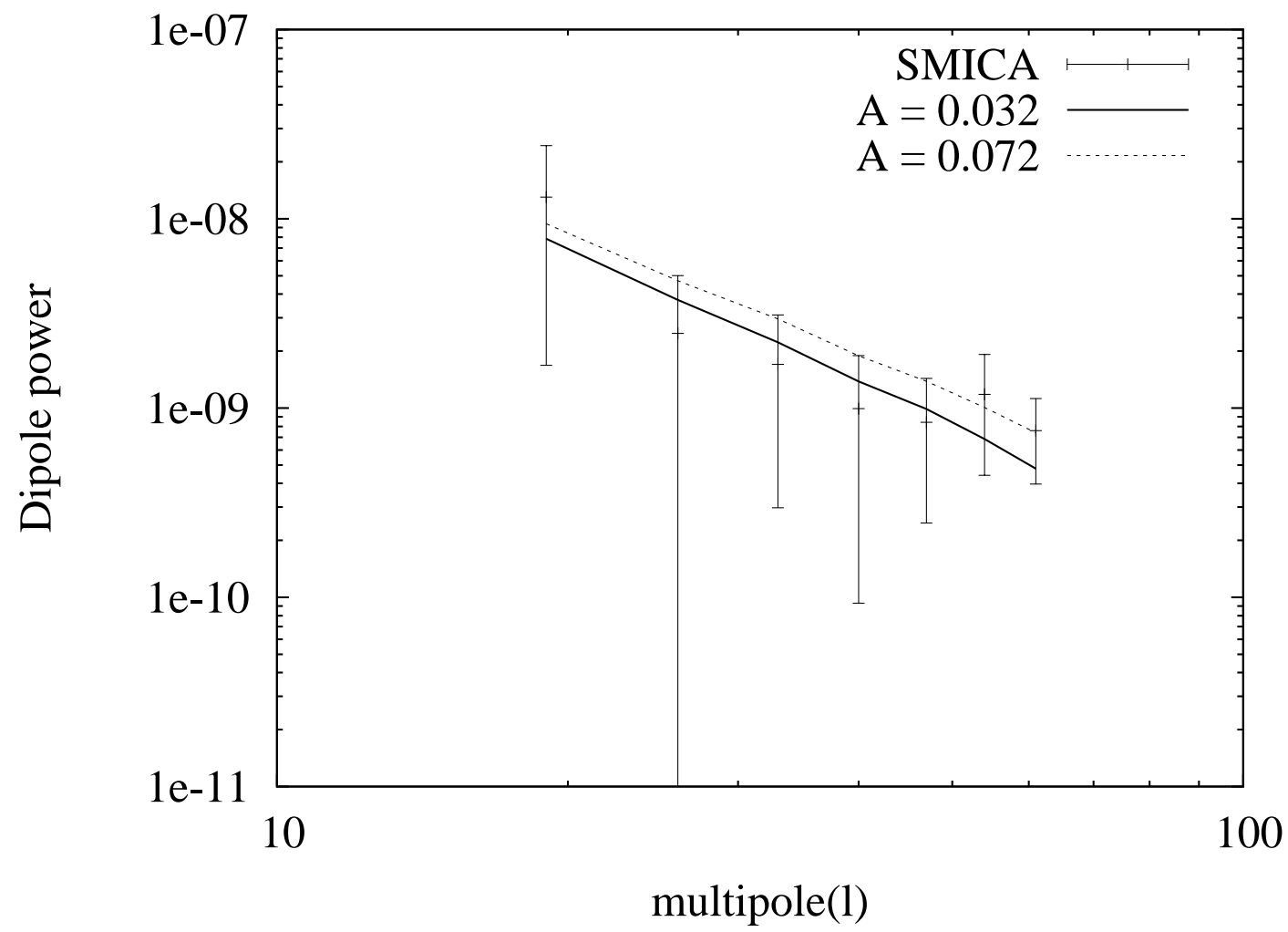

Figure 3. The dipole power, $\mathcal{C}_{1}\left(\mathrm{mK}^{4}\right)$, of the temperature square field as a function of the multipole $(l)$. The solid and dotted straight lines show the theoretical prediction based on $A=0.032$ and $A=0.072$ respectively.

to the standard measure, $l(l+1) C_{l}$, used in [7-12], this yields a lower weight for high $l$ multipoles. By eliminating a few low $l$ multipoles, we find that our measure also provides a sensitive probe of the dipole modulation model. The extracted values, both the dipole amplitude and direction are found to be in agreement, within errors, with the prediction based on multipole space hemispherical analysis. In multipole space, we show that the dipole modulation model leads to correlations between multipoles which differ by $\Delta l=1$. We define a suitable measure of this correlation, which also reveals a statistically significant signal of anisotropy. The amplitude and direction is again found to be in agreement with expectations. In multipole space, we also predict a linear dependence between the $x$ and $y$ variables defined in Eq. 2.22. This test is not found to be a sensitive probe of the signal, even after eliminating low $l$ multipoles. Nevertheless, it leads to a marginally significant result after eliminating a few low $l$ multipoles. We perform a preliminary analysis in order to test the dependence of the dipole modulation amplitude $A$ on $l$. We find that the effective value of $A$ increases with $l$ in the range $l=2-64$. Our results suggest that the hemispherical anisotropy found in [7-12] can be consistently attributed to the dipole modulation model, Eq. 1.1.

Acknowledgments: We acknowledge the use of WMAP data available from NASA's LAMBDA site(http://lambda.gsfc.nasa.gov/). Some of the results in this paper have been derived using the HEALPix [26] package.

\section{References}

[1] P. Jain and J. Ralston, Modern Physics Letters A 14, 417 (1999).

[2] D. Hutsemekers, Astronomy \& Astrophysics 332, 410 (1998).

[3] A. de Oliveira-Costa, M. Tegmark, M. Zaldarriaga, and A. Hamilton, Phys. Rev. D 69, 063516 (2004). 
[4] J. Ralston and P. Jain, International Journal of Modern Physics D 13, 1857 (2004).

[5] D. Schwarz, G. Starkman, D. Huterer, and C. Copi, Physical Review Letters 93, 221301 (2004).

[6] A. Singal, The Astrophysical Journal letters 742, L23 (2011).

[7] H. Eriksen et al., The Astrophysical Journal 605, 14 (2004).

[8] H. Eriksen et al., The Astrophysical Journal Letters 660, L81 (2007).

[9] F. Hansen et al., The Astrophysical Journal 704, 1448 (2009).

[10] A. Erickcek, S. Carroll, and M. Kamionkowski, Phys. Rev. D 78, 083012 (2008).

[11] F. Paci et al., arXiv:1301.5195 (2013).

[12] F. Schmidt and L. Hui, Phys. Rev. Lett. 110, 011301 (2013).

[13] P. A. R. Ade et al., ArXiv: 1303.5083 (2013).

[14] P. Aluri and P. Jain, Modern Physics Letters A 27, 1250014 (2012).

[15] P. Rath et al., Journal of Cosmology and Astroparticle Physics 4, 7 (2013).

[16] Z. Chang, X. Li, and S. Wang, ArXiv:1307.4542 (2013).

[17] C. Gordon, W. Hu, D. Huterer, and T. Crawford, Phys. Rev. D 72, 103002 (2005).

[18] C. Gordon, The Astrophysical Journal 656, 636 (2007).

[19] S. Prunet, J.-P. Uzan, F. Bernardeau, and T. Brunier, Phys. Rev. D 71, 083508 (2005).

[20] C. Bennett et al., Astrophys.J.Suppl. 192, 17 (2011).

[21] Z. Liu, Z. Guo, and Y. Piao, ArXiv e-prints (2013).

[22] Y. Cai, W. Zhao, and Y. Zhang, ArXiv e-prints (2013).

[23] S. Flender and S. Hotchkiss, ArXiv e-prints (2013).

[24] P. A. R. Ade et al., arXiv:1303.5062.

[25] J. Hoftuft et al., The Astrophysical Journal 699, 985 (2009).

[26] K. Goŕski et al., ApJ 622, 759 (2005). 